\title{
Influence of Lateral Growth on the Surface Pit Formation of GaN Heteroepitaxial Film Grown by MOCVD
}

\author{
Zhiyuan GAO*, Xiaowei XUE, Huimin LI, Jiangjiang LI, Li MA, Wenrong WU, Deshu ZOU
}

\author{
Microelectronic School, Beijing University of Technology, Beijing, China, 100124 \\ cross $^{\text {ref }}$ http://dx.doi.org/10.5755/j01.ms.22.2.12931
}

Received 20 August 2015; accepted 19 December 2015

\begin{abstract}
This study made an attempt to understand and control the heteroepitaxial growth of GaN from the view of the essential behaviors of crystal growth. Through a comparison of the nonpolar, polar and semipolar GaN epitaxial film, the influence of lateral growth on the surface pit formation mechanism has been investigated. For a-plane GaN, the lateral growth velocities of the less inclined $\{20-21\}$ and $\{11-22\}$ facets are approaching to the velocity of $\{10-11\}$ facet under high temperature, so that the surface pit was transformed from a triangular shape to a pentagonal one. For c-plane GaN, the size of the surface pit produced by screw dislocation is determined by the lateral growth of the pit facets. A slow lateral growth rate of the inclined facets $\{10-11\}$ compared with the vertical growth rate of (0001) facet under low V/III ratio would enlarge the pit size. For (11-22) semipolar GaN, surface pit is rarely observed, because the vertical growth velocity of $\{11-22\}$ plane is slow compared with the lateral growth rate of the inclined facets, such as $\{11-20\}$ and (0001).

Keywords: a-plane GaN, c-plane GaN, (11-22) semipolar GaN, surface pit, lateral growth.
\end{abstract}

\section{INTRODUCTION}

Lateral growth often refers to the preferential growth along certain crystal planes that perpendicular to the main growth direction due to different surface energies. It is one of the intrinsic material growth behaviors that lead to diverse crystal morphologies and structures[1]. For example, nanowire is the result of strong preferential growth along one direction and restraining growth in the lateral direction [2-4]. In the metal organic chemical vapor deposition (MOCVD) growth of $\mathrm{GaN}$ [5-7] epitaxial growth cannot be completely one by one atomic layers, island growth mode is highly preferred [6-9]; as a result, the lateral growth of these islands critically determines the physical properties of the epitaxial film. In our previous study, the influences of lateral growth of cplane $\mathrm{GaN}$ on the microstructures $[10,11]$ as well as optical properties [12] have been studied. For further study, the lateral growth behavior in different crystal geometry must be compared, in order to get a full view of the lateral growth mechanism of GaN. Since surface morphology is one of the most important factors in evaluation of crystal quality, we have studied in this paper the influence of lateral growth on the surface morphology. Different epitaxial directions have been compared, including two perpendicular directions of nonpolar GaN (a-plane) and polar GaN (c-plane), and one inclined direction of semipolar GaN ((11-22) plane). It was found that although they possess completely different surface morphologies, their pit formations were all governed by the intrinsic lateral growth nature of $\mathrm{GaN}$ crystal.

\section{EXPERIMENTAL DETAILS}

The undoped GaN samples were grown with lowtemperature GaN buffer layers by metal organic chemical

\footnotetext{
${ }^{*}$ Corresponding author. Tel.: +8613488717739; fax: +861067391641 .

E-mail address: zygao@bjut.edu.cn (Z. Gao)
}

vapor deposition (MOCVD) using a two-step growth procedure. The c-plane (0001) GaN was grown on c-plane sapphire substrate, the a-plane (11-20) GaN was grown on r-plane sapphire substrate, and the (11-22) semipolar GaN was grown on m-plane sapphire substrate. Triethylgallium (TEG) and $\mathrm{NH}_{3}$ were used as the Ga source and $\mathrm{N}$ source, respectively, and $\mathrm{H}_{2}$ was used as a carrier gas. The reactor pressure was 40 Torr, and the buffer layer temperature was $450{ }^{\circ} \mathrm{C}$. The lateral growth velocity was changed through changing temperature and V/III ratio, because these parameters determine the kinetics and thermodynamics process of MOCVD growth, such as surface diffusion, absorption and desorption, etc., and the overall effects would be reflected as the variations of surface orientationdependent growth velocity, which could be determined through the semiempirical kinetic Wulff plot, as discussed in section 4. The temperature of the high-temperature (HT) GaN layer was varied from $865{ }^{\circ} \mathrm{C}$ to $965{ }^{\circ} \mathrm{C}$, and the V/III ratio was varied from 1940 to 3160 . Each time, only one parameter was changed while the others were kept unchanged. The thickness of the $\mathrm{HT} \mathrm{GaN}$ was approximately $1 \mu \mathrm{m}$. The as-grown samples were characterized by scanning electron microscope (SEM), atomic force microscope (AFM), X-ray diffraction (XRD) and transmission electron microscopy (TEM). The TEM specimens were prepared by means of focused ion beam (FIB).

\section{RESULTS}

Fig. $1 \mathrm{a}$ is the SEM image of the surface morphology of a-plane $\mathrm{GaN}$ grown under relative high V/III ratio and relative low temperature. Large triangular surface pits bounded by the inclined $\{10-11\}$ and vertical (000-1) facets are uniformly distributed. The edge lengths are in the range of $500 \mathrm{~nm} \sim 2 \mu \mathrm{m}$ in the [1-100] direction and density is on the order of $10^{6} \mathrm{~cm}^{-2}$. 
Table 1. Variations of surface pit density and FWHM with different growth conditions of a-plane GaN

\begin{tabular}{|c|c|c|c|c|c|c|}
\hline Sample & V/III & Temperature, ${ }^{\circ} \mathrm{C}$ & Pit density, $\mathrm{cm}^{-2}$ & Pit shape & $(110) \mathrm{FWHM}^{\circ}$ & $(102) \mathrm{FWHM},{ }^{\circ}$ \\
\hline A & 2945 & 950 & $9.0 \cdot 10^{6}$ & Pentagon & 0.465 & 0.826 \\
\hline B & 2945 & 915 & $9.1 \cdot 10^{6}$ & Triangle & 0.561 & 1.013 \\
\hline C & 2945 & 865 & $9.7 \cdot 10^{6}$ & Triangle & 0.587 & 1.039 \\
\hline D & 2340 & 965 & $3.5 \cdot 10^{6}$ & Pentagon & 0.484 & 0.754 \\
\hline E & 2340 & 915 & $3.5 \cdot 10^{6}$ & Triangle & 0.671 & 0.969 \\
\hline F & 2340 & 865 & $1.0 \cdot 10^{6}$ & Triangle & 0.569 & 0.988 \\
\hline G & 1940 & 915 & $9.0 \cdot 10^{5}$ & Triangle & 0.529 & 0.844 \\
\hline
\end{tabular}

The cross-sectional TEM images in Fig. $1 \mathrm{~b}$ reveal that this type of pit is formed through island coalescing rather than defects. Under the diffraction condition of this image, the visible defects are perfect dislocations, partial dislocations (PD) and basal plane stacking fault (BSF). Dislocations show line contrast while BSF show band contrast. It can be seen that the pit does not connect with a single defect, but a group of defects, including all the visible types of defects. There is an image contrast at two sides of the inclined facets, which is produced by differing diffraction conditions in the two neighboring grains, indicating that these are two grains being twist/tilt in between. The image contrast area is indicated by the dashed frame in the figure. The grain boundary is along the diagonal of the frame. It can be seen that the top-right area is dark while the bottom left area is bight.
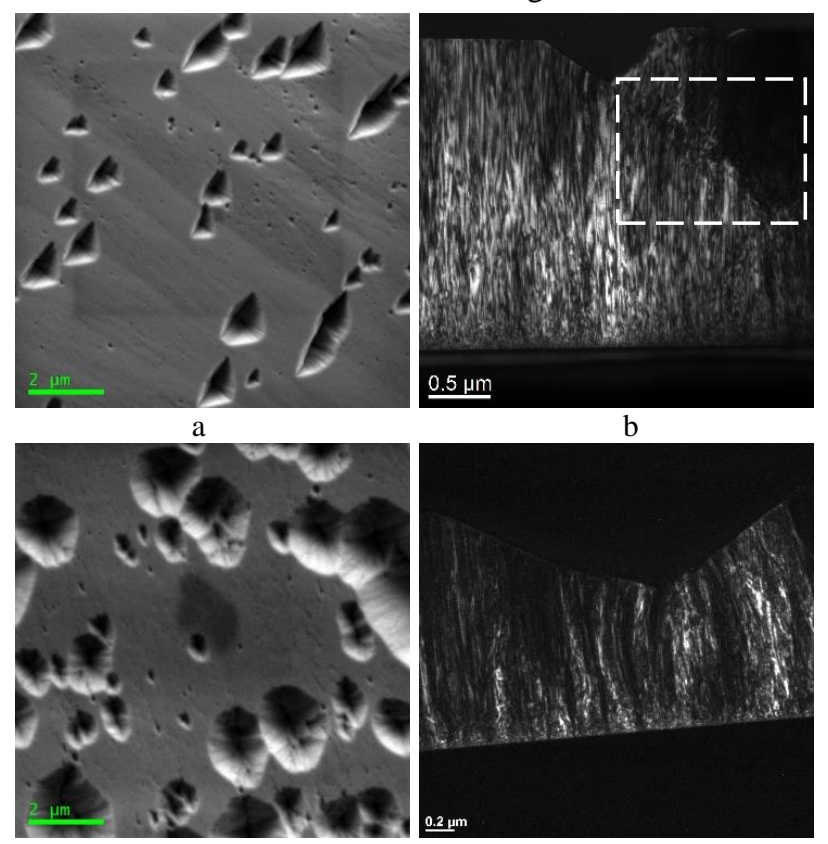

c

d

Fig. 1. a-SEM image of the surface morphology of a-plane GaN grown under relative high V/III ratio of 2945 and relative low temperature of $915^{\circ} \mathrm{C}$; b - cross-sectional weak beam dark-field TEM images of the triangular surface pit with $\mathrm{g}=10-11$ near the [01-11] zone axis; $\mathrm{c}-\mathrm{SEM}$ image of the surface morphology of a-plane $\mathrm{GaN}$ grown under relative high V/III ratio of 2945 and relative high temperature of $950{ }^{\circ} \mathrm{C} ; \mathrm{d}$-cross-sectional weak beam dark-field TEM images of the pentagonal surface pit with $\mathrm{g}=0002$ near the [1-100] zone axis

Dislocations distribute uniformly in the area with and without the pit, however, they bend when approaching to the inclined facets of the pit, because dislocations tend to intersect a free surface at $90^{\circ}$ in response to the image forces acting on the dislocation and to minimize the total dislocation line energy [17]. Table 1 summarized the variation data of the surface pit density and XRD FWHM with the growth temperature and V/III ratio of a-plane GaN. It can be seen that decreasing growth V/III ratio would diminish these surface imperfections, in consistent with the literature [13-16]. Fig. 2 a ploted the variation trend of surface pit density with V/III ratio under $915^{\circ} \mathrm{C}$. Increasing growth temperature, the a-plane $\mathrm{GaN}$ surface morphology would change from the triangular pit feature to pentagonal one, Fig. $1 \mathrm{c}$ is the SEM image of the surface morphology of a-plane GaN grown under the same V/III ratio as the sample in Fig. 1 a but relative high temperature of $950{ }^{\circ} \mathrm{C}$. The pentagonal pits are bounded by the less inclined facets of $\{10-1-2\},\{11-22\}$, and $\{20-21\}$. These pits also result from incomplete island coalescence, as directly seen from the cross-sectional TEM image in Fig. $1 \mathrm{~d}$. Under the diffraction condition of this image, the visible defects are perfect edge dislocations, mixed dislocations and PDs. It can be seen that the pit is connected with a group of defects, similar to the crosssectional image of the triangular pit. The facets that composed the pit became less inclined, and dislocations still bend toward them.

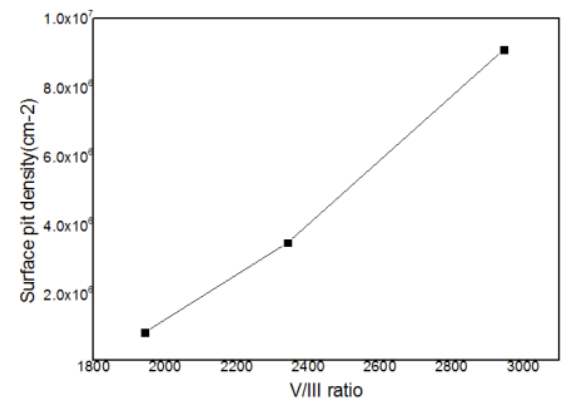

a

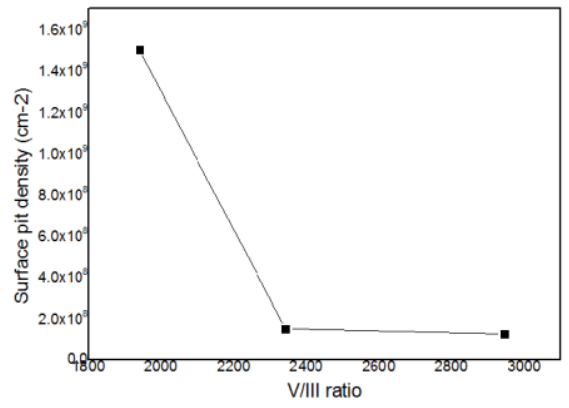

b

Fig. 2. Variation trend of surface pit density with V/III ratio under $915^{\circ} \mathrm{C}$ for: $\mathrm{a}-\mathrm{a}$-plane $\mathrm{GaN}$; $\mathrm{b}$-c-plane $\mathrm{GaN}$ 
Table 2. Variations of surface pit density and FWHM with different growth conditions of c-plane GaN

\begin{tabular}{|c|c|c|c|c|c|}
\hline Sample & V/III & Temperature, ${ }^{\circ} \mathrm{C}$ & ${\text { Pit density, } \mathrm{cm}^{-2}}^{(002)} \mathrm{FWHM}^{\circ}$ & $(102) \mathrm{FWHM}^{\circ}$ \\
\hline $\mathrm{A}$ & 3160 & 950 & $5.00 \cdot 10^{7}$ & 0.128 & 0.522 \\
\hline $\mathrm{B}$ & 2945 & 915 & $1.25 \cdot 10^{8}$ & 0.144 & 0.419 \\
\hline $\mathrm{C}$ & 2945 & 865 & $1.50 \cdot 10^{9}$ & 0.138 & 0.369 \\
\hline $\mathrm{D}$ & 2340 & 965 & $1.00 \cdot 10^{9}$ & 0.122 & 0.372 \\
\hline $\mathrm{E}$ & 2340 & 915 & $1.50 \cdot 10^{8}$ & 0.137 & 0.413 \\
\hline $\mathrm{F}$ & 2340 & 865 & $2.50 \cdot 10^{9}$ & 0.141 & 0.343 \\
\hline $\mathrm{G}$ & 1940 & 915 & $1.50 \cdot 10^{9}$ & 0.128 & 0.391 \\
\hline
\end{tabular}

The typical surface morphology of c-plane $\mathrm{GaN}$ is the stepped surface as shown in the AFM image of Fig. 3 a. Atomic surface steps intersect and end at many tiny spots, as indicated by the white arrows in the image. The density of these spots is on the order of $10^{8} \mathrm{~cm}^{-2}$. It is generally believed that these spots are the surface terminations of screw component dislocations [18-20]. In the crosssectional TEM images in Fig. $3 \mathrm{~b}$ and c, the tiny surface pit is oberved to be connected with a single dislocation line. The size of the pit is so small that it could only be seen under the enlarged view of the image, as shown in the inset of Fig. 3 b. This dislocation line is screw type, since it shows up under diffraction condition $g=0002$ in Fig. $2 \mathrm{~b}$ but disappears under $\mathrm{g}=11-20$ in Fig. $2 \mathrm{c}$ according to the invisible criteria [21].

Table 2 summarized the variation data of the surface pit density and XRD FWHM with the growth temperature and V/III ratio of c-plane GaN. Lowering growth V/III ratio, the surface pits became distinguished, as ploted Fig. $2 \mathrm{~b}$, and are enlarged as shown in the $2 \mu \mathrm{m} \times 2 \mu \mathrm{m}$ AFM image of Fig. 3 d. The size of the largest pit in the image is approaching to $100 \mathrm{~nm}$. These pits are also connected with surface steps as the same as the case of the tiny pits in Fig. 3 a, and the apex of the inverted pit is also connected with screw dislocation line determined from the cross-sectional TEM images of Fig. 3 e and f taken in the same region of the pit but using different diffraction conditions.

The surface pit density does not shows strong correlationship with the FWHM of the XRD rocking curve, which is usually used to reflect defect densities. For aplane $\mathrm{GaN}$, (110) reflection is the symmetric refelection, which diffraction peak broadening can be taken as an appropriate figure of merit for the mean tilt angle of mosaic structure induced by screw component dislocations, and the asymmetric (102) peak broadening contains the information of the twist induced by edge component dislocations. For c-plane $\mathrm{GaN}$, the symmetric (002) reflection is related to tilt caused by screw component dislocation, and the asymmetric reflection (102) contains the information of the twist produced by edge component dislocation $[22,23]$. The uncorrelation bewteen surface pit density and XRD FWHM is understandable for a-plane $\mathrm{GaN}$, since pit does not produced by defects, however, for c-plane GaN, it indicates that screw dislocation is just an inductive factor, the pit size is controlled by the same machanism as in the a-plane GaN growth. If the pit is too small to be observed, the measured pit density would be of low level. In addition, not all the dislocations can propagate to the surface, some may bend or annihilate with each other, which also deteriorates the correlationship. So if it is for an accurate evaluation of dislocation density, chemical wet etching is needed, which could expose the dislocation position clearly.

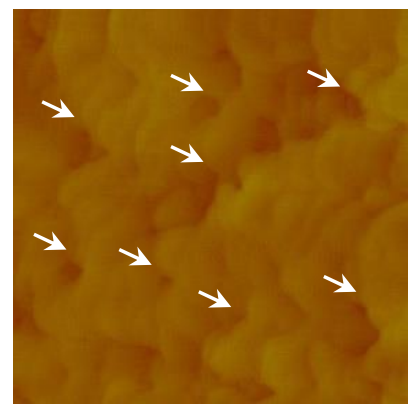

a

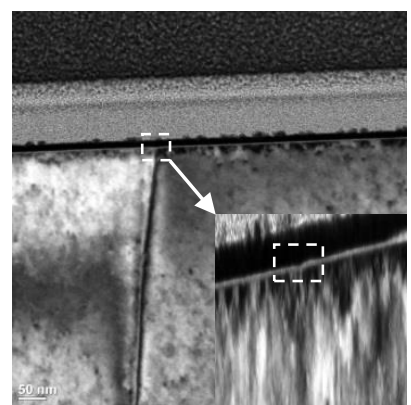

c

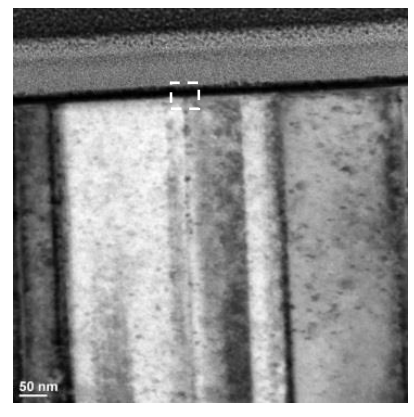

e
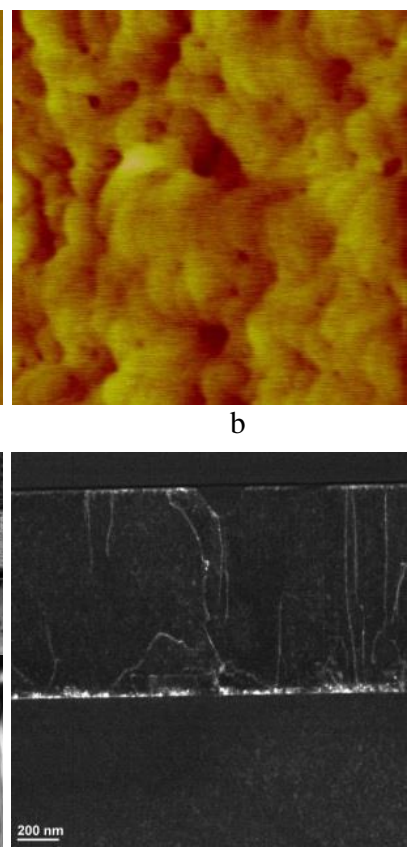

d

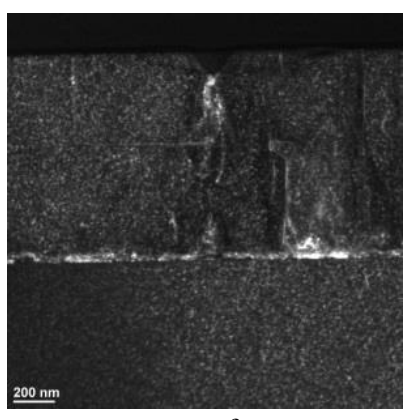

f
Fig. 3. $a-2 \mu \mathrm{m} \times 2 \mu \mathrm{m}$ AFM image of the surface morphology of c-plane GaN grown under relative high V/III ratio of 2340. The RMS roughness is $0.33 \mathrm{~nm}$. Surface pits appear as tiny spots as indicated by the white arrows in the image. Cross-sectional two beam bright-field TEM images taken in the same region of a pit but using different diffraction conditions of $\mathrm{b}-\mathrm{g}=0002 ; \mathrm{c}-\mathrm{g}=11-20$ near the [1-100] zone axis. The pit position is indicated by the white dashed frame. Inset in Fig. $3 \mathrm{~b}$ is the enlarged view of bright-filed image showing the pit; $d-2 \mu \mathrm{m} \times 2 \mu \mathrm{m}$ AFM image of the surface morphology of c-plane $\mathrm{GaN}$ grown under relative low V/III ratio of 1940. The RMS roughness is $0.35 \mathrm{~nm}$. Cross-sectional weak beam darkfield TEM images taken in the same region of a pit but using different diffraction conditions of $\mathrm{e}-\mathrm{g}=0002$; $\mathrm{f}-\mathrm{g}=11-20$ near the $[1-100]$ zone axis 


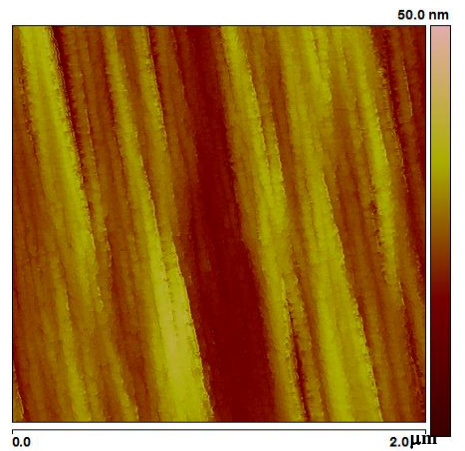

Fig. 4. $2 \mu \mathrm{m} \times 2 \mu \mathrm{m}$ AFM image of the surface morphology of (11-22) semipolar GaN. The RMS roughness is $4.7 \mathrm{~nm}$. No surface pits are observed

It is interesting to see that (11-22) semipolar GaN surface is free of pit, different from the a-plane and c-plane sample, in consistent with the literature[24]. Fig. 4 is the typical surface morphology of (11-22) GaN. Only strips were observed in the $2 \mu \mathrm{m} \times 2 \mu \mathrm{m}$ AFM sample area. Further changing the V/III or temperature would not produce a pit at all.

\section{DISCUSSION}

Under equilibrium conditions, the shape of a growing crystal in an anisotropic system can be determined by minimizing the total surface energy under mass conservation. MOCVD is an equilibrium process and as such this thermodynamic model is appropriate. However, nitrides are different because of the high growth temperature employed and the large kinetic barriers associated with the decompostion of $\mathrm{NH}_{3}$ on the surface. Consequently, the lateral growth mechanism in heteroepitaxy inherently consists of numerous atomistic processes governed by competitions between kinetics and thermodynamics. If the vapor-phase transport is sufficiently fast such that each section of the growth front is exposed to the same chemical environment, the evolution of the surface may be surface reaction limited. In this case, the morphology evolution will be determined entirely by the surface orientation-dependent growth velocity. During the island coalescence process, the fastgrowing facets grow to extinction while the slow-growing facets expand and ultimately define the island shape. The growth velocities of each facets could be obtained through the semiempirical kinetic Wulff plot [16,25], which combined the Wulff construction based on minimizing the total free energy principle under equilibrium conditions to the surface orientation-dependent growth velocity plot in the kinetically controlled growth regime.

In a-plane nonpolar $\mathrm{GaN}$ grown with the conventional two-step procedure, the incomplete lateral coalescence of nucleation islands on top of the fine crystals in the buffer would cause large inverted pyramid pits on the surface [13-16, 26-28], particularly under the growth condition of high V/III ratio. From the kinetic Wulff plot, it could be observed that under relative high V/III ratio, the ratio between lateral growth rate of the inclined facets $\{10-11\}$ and vertical growth rate of (11-20) facet is low, so that island coalescence of a-plane $\mathrm{GaN}$ is slow and inclined facets remain, resulting in the pit. Under low V/III ratio, the lateral vs. vertical growth velocity ratio is high, so that growth front along the inclined facet direction would gradually change back to the epitaxial orientation to fill up the pit. In the same way, this kinetic process also governs the shape of the pit. In some literatures, these surface pits were triangular shapes bound by one vertical (000-1) and two inclined $\{10-11\}$ facets[14], while in some studies, pentagonal shapes composed of $\{10-1-2\},\{20-21\}$, and $\{11-22\}$ facets $[15,16]$. From our experimental results, it could be seen that the pentagonal pit was transformed from the triangular one due to different lateral growth velocities of a-plane $\mathrm{GaN}$. Under high temperature, the growth velocity of $\{20-21\}$ and $\{11-22\}$ is approaching to the velocity of $\{10-11\}$ facet, so that the pit would be more likely bounded by facets of $\{20-21\},\{11-22\}$ and $\{10-1-2\}$ rather than facets of $\{10-11\}$ and (000-1). Thereafter, the triangular shaped pit transformed to a pentagonal pit.

This lateral growth mechanism also influences the facet formations of the surface pits in c-plane GaN. It is generally accepted that the formation of the small pits on c-plane $\mathrm{GaN}$ surface is due to the tension strain of screw dislocations[18-20], however, it deserves further consideration that the pit size would not vary significantly as it did in the a-plane GaN, since the strain energy of the screw dislocation should remain the same in samples grown under different conditions. It indicates that the pit is influenced by the kinetic process of the lateral growth of the facets that composed the pit. Dislocation produced a pit on the surface, but the lateral growth of the pit facets determines its relative size. Under low V/III ratio, the ratio between the lateral growth rate of the inclined facets $\{10$ $11\}$ and the vertical growth rate of (0001) facet is low, so that the pit would be difficult to be filled, and at the end of growth, it remains on the surface with relative large size.

For (11-22) semipolar GaN, pit is rarely observed on the surface[24], which could be understood by the relative fast lateral growth. The inclined facet (11-22) becomes the free surface and (11-20) or (0001) facet becomes the inclined facet. Because the growth velocity of $\{11-22\}$ plane is slow compared with $\{11-20\}$ and (0001), the lateral growth rate of the nucleation islands is fast enough to result in a pit free surface. Changing the V/III ratio or temperature would not produce surface pit, owing to the intrinsic preferential lateral growth behavior of this epitaxial orientation growth.

\section{CONCLUSIONS}

The lateral growth mechanism in heteroepitaxial growth of $\mathrm{GaN}$ critically influences the surface morphology evolution in MOCVD. A relative slow lateral growth of the inclined facets compared with the vertical growth along the epitaxial orientation will result in a pitted surface for a-plane $\mathrm{GaN}$ and even transform the pit shape, and for c-plane $\mathrm{GaN}$, this lateral growth mechanism will also enlarge size of the small surface pits which is produced by screw dislocations. In addition, since the lateral vs. vertical growth rate is fast, the (11-22) semipolar $\mathrm{GaN}$ is free of surface pit.

\section{Acknowledgement}

This work was supported by National Natural Science Foundation of China (Grant No. 11204009), and Beijing 
Municipal Natural Science Foundation (Grant No.4142005).

\section{REFERENCES}

1. Zhong, W.Z. Crystal Growth Morphology, Science Press, Beijing, 1999: pp. 395-396.

2. Kesaria, M., Shetty, S., Shivaprasad, S.M. Evidence for Dislocation Induced Spontaneous Formation of GaN Nanowalls and Nanocolumns on bare c-plane Sapphire Crystal Growth Design 11 2011: pp. 4900-4903. http://dx.doi.org/10.1021/cg200749w

3. Masala, O., Seshadri, R. Synthesis Routes for Large Volumes of Nanoparticles Annual Review of Material Research 34 2004: pp. $41-81$.

4. Arpan, D., Nagaraja, K.K., $\quad$ Malleswararao, T., Shivaprasad, S.M. Spontaneous Growth of InGaN Nanostructures Directly on C-plane Sapphire by Plasma Assisted Molecular Beam Epitaxy Material Research Express 1 2014: pp. 035019-1-035019-5.

5. Xu, S.R., Lin, Z.X., Xue, X.Y., Liu, Z.Y., Ma, J.C., Jang, T., Mao, W., Wang, D.H., Zhang, J.C., Hao, Y. Comparative Study on Characteristics of Basal Plane Stacking Faults of Nonpolar a-Plane and Semipolar (11-22) GaN Chinese Physics Letters 29 2012: pp. 017803-1-017803-3.

6. Grzegorczyk, A.P., Hageman, P.R., Weyher, J.L. Influence of Sapphire Annealing in Trimethylgallium atmosphere on GaN Epitaxy by MOCVD Journal of Crystal Growth 283 2005: pp. $72-80$.

http://dx.doi.org/10.1016/j.jcrysgro.2005.05.072

7. Wang, C.M., Wang, X.L., Hu, G.X. The effect of AlN Growth Time on the Electrical Properties of Al0.38Ga0.62N/AlN/GaN HEMT Structures Journal Crystal Growth 289 2006: pp. $415-418$. http://dx.doi.org/10.1016/j.jcrysgro.2005.11.118

8. Qian, W., Skowronski, M., Graef, D. Microstructural Characterization of GaN films Grown on Sapphire by Organometallic Vapor Phase Epitaxy Applied Physics Letters 66 1995: pp. $1252-1254$.

9. Moran, B., Wu, F., Romanov, A.E. Structural and Morphological Evolution of GaN Grown by Metalorganic Chemical Vapor Deposition on SiC substrates Journal of Crystal Growth 273 2004: pp. 38-42. http://dx.doi.org/10.1016/j.jcrysgro.2004.08.012

10. Gao, Z.Y., Duan, H.T., Hao, Y., Li, P.X., Zhang, J.F. Formation and Optical Properties of the Large V-shaped Surface Pits in GaN Thin Film Chinese Journal of Material Research 22 2008: pp. 657-663.

11. Gao, Z.Y., Hao, Y., Li, P.X., Zhang, J.C. Role of Lateral Growth on the High Temperature GaN layer China Technological Sciences 39 2009: pp. 124-128.

12. Gao, Z.Y., Hao, Y., Li, P.X., Zhang, J.C. Influence of Threading Dislocations on the Luminescence Efficiency Journal of Semiconductors 29 2008: pp. 522-525.

13. Zhang, J., Tian, W., Wu, F., Wan, Q., Wang, Z., Zhang, J., Li, Y., Dai, J., Fang, Y., Wu, Z., Chen, C., Xu, J., Li, X. The Effects of Substrate Nitridation on the Growth of Nonpolar aplane GaN on r-plane Sapphire by MOCVD Applied Surface Science 307 2014: pp. 525-532. http://dx.doi.org/10.1016/j.apsusc.2014.04.069

14. Song, K.M., Kim, J.M., Lee, D.H., Shin, C.S., Ko, C.G., Kong, B.H., Cho, H.K., Yoon, D.H. Growth Behavior and Growth rate Dependency in LEDs Performance for Mg-doped a-plane GaN Journal of Crystal Growth 326 2011: pp. $135-139$. http://dx.doi.org/10.1016/j.jcrysgro.2011.01.083

15. Badcock, T.J., Hao, R., Moram, M.A., Dawson, P., Kappers, M.J., Humphreys, C.J. Properties of Surface Pit Related Emission in a-plane InGaN/GaN Quantum Wells Grown on r-plane Sapphire Physica Status Solidi C 8 2011: pp. $2179-2181$. http://dx.doi.org/10.1002/pssc.201001043

16. Sun, Q., Yerino, C.D., Ko, T.S., Cho, Y.S., Lee, I.H., Han, J., Coltrin, M.E. Understanding Nonpolar GaN Growth Through Kinetic Wulff plots Journal of Applied Physics 104 2008: pp. 093523-1-093523-5.

17. Hao, R., Kappers, M.J., Moram, M.A., Humphreys, C.J. Defect Reduction Process in Heteroepitaxial Non-polar a-plane GaN Films Journal of Crystal Growth 337 2011: pp. $81-86$. http://dx.doi.org/10.1016/j.jcrysgro.2011.10.004

18. Miraglia, P.Q., Preble, E.A., Roskowski, A.M., Infeldt, S., Davis, R.F. Helical-type Surface Defects in GaN Thin Films Epitaxially Grown on GaN Templates at Reduced Temperatures Journal of Crystal Growth 253 2003: pp. 16-25.

19. Son, K.S., Kim, D.G., Cho, H.K., Lee, K., Kim, S. Park, K. Formation of V-shaped Pits in GaN Thin Films Grown on High Temperature GaN Journal of Crystal Growth 261 2004: pp. $50-54$. http://dx.doi.org/10.1016/j.jcrysgro.2003.08.075

20. Heying, B., Tarsa, E.J., Elsass, C.R., Fini, P., DenBaars, S.P., Speck, J.S. Dislocation Mediated Surface Morphology of GaN Journal of Applied Physics 85 1999: pp. 6470-6476. http://dx.doi.org/10.1063/1.370150

21. Kong, B.H., Sun, Q., Han, J., Lee, I.H., Cho, H.K. Classification of Stacking Faults and Dislocations Observed in Nonpolar a-plane GaN Epilayers using TEM Applied Surface Science 258 2012: pp. 2522-2528. http://dx.doi.org/10.1016/j.apsusc.2011.10.086

22. Chierchia R., Bottcher, T., Heinke, H., Einfeldt, S., Figge, S., Hommel, D. Microstructure of Heteroepitaxial GaN Revealed by X-Ray Diffraction Journal of Applied physics 93 (11) 2003: pp. $8918-8925$. http://dx.doi.org/10.1063/1.1571217

23. Heying, B., Wu, X.H., Keller, S., Li, Y., Kapolnek, D., Keller, B.P., DenBaars, S.P., Speck, J.S. Role of Threading Dislocation Structure on the X-Ray Diffraction Peak Widths in Epitaxial GaN Films Applied Physics Letters 68 (5) 1996: pp. $643-645$. http://dx.doi.org/10.1063/1.116495

24. Xu, S.R., Hao, Y., Zhang, J.C., Cao, Y.R., Zhou, X.W., Yang, L.A., Ou, X.X., Chen, K., Mao, W. Polar Dependence of Impurity Incorporation and Yellow Luminescence in $\mathrm{GaN}$ films Grown by MOCVD Journal of Crystal Growth 312 2010: pp. $3521-3524$. http://dx.doi.org/10.1016/j.jcrysgro.2010.09.026

25. Sun, Q., Yerino, C.D., Leung, B., Han, J., Coltrin, M.E. Understanding and Controlling Heteroepitaxy with the Kinetic Wulff Plot: A case study with GaN Journal of Applied Physics 110 2011: pp. 053517-1-053517-10.

26. Hollander, J.L., Kappers, M.J., $\quad$ McAleese, C., Humphreys, C.J. Improvements in a-plane GaN Crystal Quality by a Two-Step Growth Process Applied Physics Letters 92 2008: pp. 101104-1-101104-3.

27. Tian, Y., Dai, J.N., Xiong, H., Zheng, G., $\quad$ Ryu, M., Fang, Y.Y., Chen, C.Q. Effects of the V/III Ratio of a LowTemperature GaN Buffer Layers on the Structural and Optical Properties of a-GaN Films Grown on r-Plane Sapphire Substrates by MOCVD Chinese Physics Letters 29 (8) 2012: pp. 088101-1-088101-4.

28. Wang, J.X., Wang, L.S., Yang, S.Y., Li, H.J., Zhao, G.J., Zhang, H., Wei, H.Y., Jiao, C.M., Zhu, Q.S., Wang, Z.G. Effects of V/III Ratio on a-plane GaN Epilayers with an InGaN Interlayer Chinese Physics $B \quad 23$ (2) 2014: pp. 026801-1-026801-5. 